\title{
QUENCHED CENTRAL LIMIT THEOREM FOR RANDOM WALKS IN DOUBLY STOCHASTIC RANDOM ENVIRONMENT ${ }^{1}$
}

\author{
BY BÁLINT TÓTH \\ University of Bristol and Rényi Institute, Budapest
}

\begin{abstract}
We prove the quenched version of the central limit theorem for the displacement of a random walk in doubly stochastic random environment, under the $H_{-1}$-condition, with slightly stronger, $\mathcal{L}^{2+\varepsilon}$ (rather than $\mathcal{L}^{2}$ ) integrability condition on the stream tensor. On the way we extend Nash's moment bound to the nonreversible, divergence-free drift case, with unbounded $\left(\mathcal{L}^{2+\varepsilon}\right)$ stream tensor. This paper is a sequel of [Ann. Probab. 45 (2017) 4307-4347] and relies on technical results quoted from there.
\end{abstract}

1. Introduction. Let $\left(\Omega, \mathcal{F}, \pi, \tau_{z}: z \in \mathbb{Z}^{d}\right)$ be a probability space with an ergodic $\mathbb{Z}^{d}$-action. Denote by $\mathcal{E}:=\left\{k \in \mathbb{Z}^{d}:|k|=1\right\}$ the set of possible steps of a nearest-neighbour walk on $\mathbb{Z}^{d}$. Let $p_{k}: \Omega \rightarrow\left[0, s^{*}\right], k \in \mathcal{E}$, be bounded measurable functions ( $s^{*}<\infty$ is their common upper bound). These will be the jump rates of the RWRE considered [see (2) below] and assume they are doubly stochastic,

$$
\sum_{k \in \mathcal{E}} p_{k}(\omega)=\sum_{k \in \mathcal{E}} p_{-k}\left(\tau_{k} \omega\right) .
$$

The physical meaning of (1) is that the local drift field of the walk is divergencefree, that is, the stream field of an incompressible flow in stationary regime.

Given these, define the continuous time nearest neighbour random walk $t \mapsto$ $X(t) \in \mathbb{Z}^{d}$ as a Markov process on $\mathbb{Z}^{d}$, with $X(0)=0$ and conditional jump rates

$$
\mathbf{P}_{\omega}(X(t+d t)=x+k \mid X(t)=x)=p_{k}\left(\tau_{x} \omega\right) d t+o(d t),
$$

where the subscript $\omega$ denotes that the random walk $X(t)$ is a Markov process on $\mathbb{Z}^{d}$ conditionally, with fixed $\omega \in \Omega$, sampled according to $\pi$. The continuous time setup is for convenience only. Since the jump rates are bounded, this is fully equivalent with a discrete time walk.

We will use the notation $\mathbf{P}_{\omega}(\cdot)$ and $\mathbf{E}_{\omega}(\cdot)$ for quenched probability and expectation. That is, probability and expectation with respect to the distribution of the

Received October 2017; revised December 2017.

${ }^{1}$ Supported by EPSRC (UK) Fellowship EP/P003656/1, by The Leverhulme Trust (UK) through the International Network Laplacians, Random Walks, Quantum Spin Systems and by OTKA (HU) K-109684.

MSC2010 subject classifications. 60F05, 60G99, 60K37.

Key words and phrases. Random walk in random environment, quenched central limit theorem, Nash bounds. 
random walk $X(t)$, conditionally, with given fixed environment $\omega$. The notation $\mathbf{P}(\cdot):=\int_{\Omega} \mathbf{P}_{\omega}(\cdot) d \pi(\omega)$ and $\mathbf{E}(\cdot):=\int_{\Omega} \mathbf{E}_{\omega}(\cdot) d \pi(\omega)$ will be reserved for annealed probability and expectation. That is, probability and expectation with respect to the random walk trajectory $X(t)$ and the environment $\omega$, sampled according to the distribution $\pi$.

It is well known (and easy to check, see, e.g., [17]) that due to double stochasticity (1) the annealed set-up is stationary and ergodic in time: the process of the environment as seen from the position of the random walker

$$
\eta(t):=\tau_{X(t)} \omega
$$

is a stationary and ergodic Markov process on $(\Omega, \pi)$ and consequently the random walk $t \mapsto X(t)$ will have stationary and ergodic annealed increments. $\mathbb{R}^{d}$

Next we define, for $k \in \mathcal{E}, s_{k}: \Omega \rightarrow\left[0, s^{*}\right], v_{k}: \Omega \rightarrow\left[-s^{*}, s^{*}\right]$, and $\psi, \varphi: \Omega \rightarrow$

$$
\begin{aligned}
s_{k}(\omega):=\frac{p_{k}(\omega)+p_{-k}\left(\tau_{k} \omega\right)}{2}, & \psi(\omega):=\sum_{k \in \mathcal{E}} k s_{k}(\omega), \\
v_{k}(\omega):=\frac{p_{k}(\omega)-p_{-k}\left(\tau_{k} \omega\right)}{2}, & \varphi(\omega):=\sum_{k \in \mathcal{E}} k v_{k}(\omega) .
\end{aligned}
$$

The local quenched drift of the random walk is

$$
\mathbf{E}_{\omega}(d X(t) \mid X(t)=x)=\left(\psi\left(\tau_{x} \omega\right)+\varphi\left(\tau_{x} \omega\right)\right) d t+o(d t) .
$$

Note that from the definitions (4) it follows that for $\pi$-almost all $\omega \in \Omega$

$$
\begin{aligned}
s_{k}(\omega)-s_{-k}\left(\tau_{k} \omega\right) & =0, & \psi_{i}(\omega) & =s_{e_{i}}(\omega)-s_{e_{i}}\left(\tau_{-e_{i}} \omega\right), \\
v_{k}(\omega)+v_{-k}\left(\tau_{k} \omega\right) & =0, & \varphi_{i}(\omega) & =v_{e_{i}}(\omega)+v_{e_{i}}\left(\tau_{-e_{i}} \omega\right) .
\end{aligned}
$$

In addition, condition (1) is equivalent to

$$
\sum_{k \in \mathcal{E}} v_{k}(\omega) \equiv 0, \quad \pi \text {-a.s. }
$$

Thus, $\left(v_{k}\left(\tau_{x} \omega\right)\right)_{k \in \mathcal{E}, x \in \mathbb{Z}^{d}}$ is a stationary sourceless (or divergence-free) flow on the lattice $\mathbb{Z}^{d}$. The physical interpretation of the divergence-free condition (6) is that the walk (2) models the motion of a particle suspended in stationary, incompressible flow, with thermal noise.

In order that the walk $t \mapsto X(t)$ have zero annealed mean drift, we also assume that for all $k \in \mathcal{E}$

$$
\int_{\Omega} v_{k}(\omega) d \pi(\omega)=0
$$

Our next assumption is the strong ellipticity condition for the symmetric part of the jump rates: there exists another positive constant $s_{*} \in\left(0, s^{*}\right]$ such that for $\pi$-almost all $\omega \in \Omega$ and all $k \in \mathcal{E}$

$$
s_{k}(\omega) \geq s_{*}, \quad \pi \text {-a.s. }
$$


Note that the ellipticity condition is imposed only on the symmetric part $s_{k}$ of the jump rates and not on the jump rates $p_{k}$. It may happen that $\pi\left(\left\{\omega: \min _{k \in \mathcal{E}} p_{k}(\omega)=\right.\right.$ $0\})>0$, as it is the case in some examples given in [19].

By applying a linear time change we may and will choose $s_{*}=1 \leq s^{*}<\infty$.

Finally, we formulate the notorious $H_{-1}$-condition which plays a key role. Denote for $i, j=1, \ldots, d$,

$$
C_{i j}(x):=\int_{\Omega} \varphi_{i}(\omega) \varphi_{j}\left(\tau_{x} \omega\right) d \pi(\omega), \quad \widehat{C}_{i j}(p):=\sum_{x \in \mathbb{Z}^{d}} e^{\sqrt{-1} x \cdot p} C_{i j}(x) .
$$

By Bochner's theorem, the Fourier transform $\widehat{C}$ is positive definite $d \times d$-matrixvalued-measure on $[-\pi, \pi)^{d}$. The no-drift condition (7) is equivalent to $\widehat{C}_{i j}(\{0\})=$ 0 , for all $i, j=1, \ldots, d$. With slight abuse of notation we denote this measure formally as $\widehat{C}_{i j}(p) d p$ even though it could be not absolutely continuous with respect to Lebesgue.

The $H_{-1}$-condition is the following:

$$
\int_{[-\pi, \pi)^{d}}\left(\sum_{j=1}^{d}\left(1-\cos p_{j}\right)\right)^{-1} \sum_{i=1}^{d} \widehat{C}_{i i}(p) d p<\infty .
$$

This is an infrared bound on the correlations of the skew-symmetric part of the drift field, $x \mapsto \varphi\left(\tau_{x} \omega\right) \in \mathbb{R}^{d}$. It implies diffusive upper bound on the annealed walk [see the upper bound in (KT28)] and turns out to be a natural sufficient condition for the diffusive scaling limit (i.e., CLT for the annealed walk); see Theorem 1 in [19]. [Throughout this note, (KTxx) points at display number (xx) in [19].] Three other equivalent formulations of (9) are given in [19]. Two of these, (KT35) and Proposition 4(ii) of [19] are of particular interest, since we shall use them. Note that the $H_{-1}$-condition (9) actually formally implies the no-drift condition (7).

It is proved in Proposition 4(ii) of [19] that the $H_{-1}$-condition (9) is equivalent to the existence of a stationary and square integrable stream-tensor-field whose curl (or rotation) is exactly the source-less (divergence-free) flow $v$. More explicitly, there exist $h_{k, l} \in \mathcal{H}, k, l \in \mathcal{E}$, with symmetries

$$
h_{k, l}(\omega)=-h_{-k, l}\left(\tau_{k} \omega\right)=-h_{k,-l}\left(\tau_{l} \omega\right)=-h_{l, k}(\omega), \quad \pi \text {-a.s. }
$$

such that

$$
v_{k}(\omega)=\sum_{l \in \mathcal{E}} h_{k, l}(\omega)
$$

Remarks on the stream tensor $h$. The fact that $v$ is expressed as in (11) with $h$ having the symmetries (10) is essentially the lattice-version of Helmholtz's theorem. In its most common three-dimensional formulation: "A divergence free vector field is the curl of a vector field." (In classical electrodynamics, this is called the vector potential.) Note that (10) means that the stream tensor field $x \mapsto h\left(\tau_{x} \omega\right)$ is actually function of the oriented plaquettes of $\mathbb{Z}^{d}$. In particular, in two-dimensions 
$x \mapsto h\left(\tau_{x} \omega\right)$ defines a stationary height function on the dual lattice $\mathbb{Z}^{2}+(1 / 2,1 / 2)$, in three-dimensions $x \mapsto h\left(\tau_{x} \omega\right)$ defines a stationary oriented flow (i.e., a vector field) on the dual lattice $\mathbb{Z}^{3}+(1 / 2,1 / 2,1 / 2)$. For more details about the stream tensor and the derivation of (10)-(11), see Section 5 of [19].

We will now assume that the stream-tensor-field has the stronger integrability,

$$
h \in \mathcal{L}^{2+\varepsilon}(\Omega, \pi),
$$

for some $\varepsilon>0$, rather than being merely square integrable. This stronger integrability condition is needed in the proof of quenched tightness of the diffusively scaled displacement $t^{-1 / 2} X(t)$. We denote

$$
h^{*}=h^{*}(\varepsilon):=\sum_{k, l \in \mathcal{E}}\left(\int_{\Omega}\left|h_{k, l}\right|^{2+\varepsilon} d \pi\right)^{1 /(2+\varepsilon)}<\infty .
$$

In [19], it was shown that for a RWRE (2) whose environment satisfies conditions (1), (8) and (9) the central limit theorem holds, under diffusive scaling and Gaussian limit with finite and nondegenerate asymptotic covariance, in probability with respect to the environment; see Theorem 1 in [19]. The proof consists of an efficient martingale approximation in the spirit of Kipnis-Varadhan, based on the relaxed sector condition introduced in [14] and down-to-earth explicit functional analysis in and over the Hilbert spaces of scalars $(\mathcal{H})$ and gradients $(\mathcal{G})$ :

$$
\begin{aligned}
\mathcal{H}:= & \left\{f \in \mathcal{L}^{2}(\Omega, \pi): \int_{\Omega} f(\omega) d \pi(\omega)=0\right\}, \\
\mathcal{G}:= & \left\{g=\left(g_{k}\right)_{k \in \mathcal{E}} \in \bigoplus_{k \in \mathcal{E}} \mathcal{H}: g_{k}(\omega)+g_{-k}\left(\tau_{k} \omega\right)=0,\right. \\
& \left.g_{k}(\omega)+g_{l}\left(\tau_{k} \omega\right)=g_{l}(\omega)+g_{k}\left(\tau_{l} \omega\right), k, l \in \mathcal{E}\right\} .
\end{aligned}
$$

We stress that in [19] $h \in \mathcal{L}^{2}(\Omega, \pi)$ is assumed, rather than (12).

The main result of the present paper is, that under conditions (1), (8), (9) and (12) [which is a marginally stronger version of (9)] actually the quenched CLT holds, with deterministic nondegenerate covariance matrix. This is Theorem 1 below.

For general background on RWRE and in particular on the quenched versus annealed CLT dichotomy, see the surveys [5, 21, 28]. For more background on random walks in doubly stochastic random environment, for interesting examples and in general more illuminating comments, see [19] and [27].

2. Results. Throughout the paper, conditions (1), (8) and (9) are assumed. [Recall that (7) is formally implied by (9), so we do not state it as a separate condition.] Propositions 2 and 3 are valid under these conditions. In Proposition 1, and as a consequence, in Proposition 4 and Theorem 1 the stronger integrability condition (12) of the stream-tensor-field is also assumed. 
PROpOSITION 1. Conditions (1), (8), (9), (12) are assumed. There exists a constant $M^{*}=M^{*}\left(d, s_{*}, s^{*}, \varepsilon, h^{*}\right)<\infty$ such that for $\pi$-almost all $\omega$,

$$
\varlimsup_{t \rightarrow \infty} t^{-1 / 2} \mathbf{E}_{\omega}(|X(t)|) \leq M^{*}
$$

In particular the scaled displacements $t^{-1 / 2} X(t)$ are quenched tight.

In the next proposition, $\Delta$ denotes the Laplacian operator acting on the Hilbert space $\mathcal{H}$, as defined in (31) below. Note that $\Delta$ is bounded, self-adjoint and negative. Thus, the operators $|\Delta|^{1 / 2}$ and $|\Delta|^{-1 / 2}$ are defined in terms of the spectral theorem. The unbounded operator $|\Delta|^{-1 / 2}$ is defined on the domain

$$
\begin{aligned}
\mathcal{H}_{-1} & :=\left\{\phi \in \mathcal{H}: \lim _{\lambda \searrow 0}\left(\phi,(\lambda I-\Delta)^{-1} \phi\right)_{\mathcal{H}}<\infty\right\} \\
& =\operatorname{Ran}|\Delta|^{1 / 2}=\operatorname{Dom}|\Delta|^{-1 / 2} .
\end{aligned}
$$

Proposition 2. Conditions (1), (8), (9) are assumed. For any $\phi \in \mathcal{H}_{-1}$ there exists a unique solution $\theta \in \mathcal{G}$ of the equation

$$
\sum_{k \in \mathcal{E}} p_{k}(\omega) \theta_{k}(\omega)=\phi(\omega)
$$

We denote by $\Theta: \Omega \times \mathbb{Z}^{d} \rightarrow \mathbb{R}$ the cocycle to which $\theta$ is the gradient: for $x \in \mathbb{Z}^{d}$ and $k \in \mathcal{E}$,

$$
\Theta(\omega, 0)=0, \quad \Theta(\omega, x+k)-\Theta(\omega, x)=\theta_{k}\left(\tau_{x} \omega\right), \quad \pi \text {-a.s. }
$$

Equations (15) and (16) amount to the fact that for all $x \in \mathbb{Z}^{d}$,

$$
\phi\left(\tau_{x} \omega\right)-\sum_{k \in \mathcal{E}} p_{k}\left(\tau_{x} \omega\right)(\Theta(\omega, x+k)-\Theta(\omega, x))=0, \quad \pi \text {-a.s. }
$$

Hence, it follows that for $\pi$-a.a. $\omega \in \Omega$ fixed, the process

$$
t \mapsto Y(t):=\int_{0}^{t} \phi\left(\tau_{X(s)} \omega\right) d s-\Theta(\omega, X(t))
$$

is a martingale in the quenched filtration

$$
\mathcal{F}_{t}:=\mathcal{F} \vee \sigma\{X(s): 0 \leq s<t\}
$$

That is, with $\omega \in \Omega$ fixed.

Due to the martingale central limit theorem and stationarity and ergodicity of the environment process $t \mapsto \eta(t)$ defined in (3) (see Section 1.2 of [19]), the $\pi$ a.s. (quenched) central limit theorem follows for the process $t \mapsto Y(t)$. 
Proposition 3. Conditions (1), (8), (9) are assumed. Let $\phi \in \mathcal{H}_{-1}$. For $\pi$ a.a. $\omega \in \Omega$, and any bounded and continuous function $f: \mathbb{R} \rightarrow \mathbb{R}$,

$$
\lim _{t \rightarrow \infty} \mathbf{E}_{\omega}\left(f\left(t^{-1 / 2} Y(t)\right)\right)=\frac{1}{\sqrt{2 \pi} \bar{\sigma}} \int_{-\infty}^{\infty} e^{-y^{2} /\left(2 \bar{\sigma}^{2}\right)} f(y) d y,
$$

with variance

$$
\bar{\sigma}^{2}:=\sum_{k \in \mathcal{E}} \int_{\Omega} s_{k}(\omega) \theta_{k}(\omega)^{2} d \pi(\omega)>0 .
$$

As a corollary of Proposition 3 we get the quenched CLT for the harmonic coordinates (i.e., the appropriately corrected displacement) of the random walker. Indeed, first note that due to the first line in (5) $\psi \in \mathcal{H}_{-1}^{d}$ holds a priori, and due to the $H_{-1}$-condition (9) $\varphi \in \mathcal{H}_{-1}^{d}$. Actually this latter fact is one of the equivalent forms of the $H_{-1}$-condition; see (KT35). (Hence the term " $H_{-1}$-condition.") Therefore, we can choose

$$
\phi=\phi^{*}:=\varphi+\psi \in \mathcal{H}_{-1}^{d}
$$

and solve (coordinate-wise) equation (15) with $\phi_{i}^{*}, i=1, \ldots, d$, on the right-hand side. We denote the solution $\theta^{*} \in \mathcal{G}^{d}$ and define the $\mathbb{R}^{d}$-valued cocycle $\Theta^{*}$ by (16), with $\theta^{*}$ as gradient. Now, let

$$
Y^{*}(t):=X(t)-\Theta^{*}(\omega, X(t)) .
$$

COROllary 1. Conditions (1), (8), (9) are assumed. For $\pi$-a.a. $\omega \in \Omega$, and any bounded and continuous function $f: \mathbb{R}^{d} \rightarrow \mathbb{R}$,

$$
\lim _{t \rightarrow \infty} \mathbf{E}_{\omega}\left(f\left(t^{-1 / 2} Y^{*}(t)\right)\right)=\left(2 \pi \operatorname{det} \bar{\sigma}^{2}\right)^{-d / 2} \int_{\mathbb{R}^{d}} e^{-\frac{1}{2} y \cdot \bar{\sigma}^{-2} y} f(y) d y,
$$

with nondegenerate covariance matrix

$$
\bar{\sigma}_{i j}^{2}:=\sum_{k \in \mathcal{E}} \int_{\Omega} s_{k}(\omega)\left(\theta_{k}(\omega)-k\right)_{i}\left(\theta_{k}(\omega)-k\right)_{j} d \pi(\omega) .
$$

The quenched CLT with the correcting terms $\Theta(X(t))$ removed will follow from Proposition 3/Corollary 1 and the following error estimate.

Proposition 4. Conditions (1), (8), (9), (12) are assumed. Let $\Omega \times \mathbb{Z}^{d} \ni$ $(\omega, x) \mapsto \Psi(\omega, x) \in \mathbb{R}$ be a square integrable zero-mean cocycle. For $\pi$-a.a. $\omega \in$ $\Omega$ and any $\delta>0$,

$$
\lim _{t \rightarrow \infty} \mathbf{P}_{\omega}(|\Psi(X(t))|>\delta \sqrt{t})=0 .
$$

Indeed, Propositions 3/Corollary 1 and Proposition 4 readily imply the main result of this paper. 
THEOREM 1. Conditions (1), (8), (9), (12) are assumed. For $\pi$-a.a. $\omega \in \Omega$, the following quenched CLTs hold:

(i) Let $\phi \in \mathcal{H}_{-1}$. For any bounded and continuous function $f: \mathbb{R} \rightarrow \mathbb{R}$,

$$
\lim _{t \rightarrow \infty} \mathbf{E}_{\omega}\left(f\left(t^{-1 / 2} \int_{0}^{t} \phi(\eta(s)) d s\right)\right)=\frac{1}{2 \pi \bar{\sigma}} \int_{-\infty}^{\infty} e^{-y^{2} /\left(2 \bar{\sigma}^{2}\right)} f(y) d y,
$$

with the variance $\bar{\sigma}^{2}$ given in (18).

(ii) For any bounded continuous function $f: \mathbb{R}^{d} \rightarrow \mathbb{R}$,

$$
\lim _{t \rightarrow \infty} \mathbf{E}_{\omega}\left(f\left(t^{-1 / 2} X(t)\right)\right)=\left(2 \pi \operatorname{det} \bar{\sigma}^{2}\right)^{-d / 2} \int_{\mathbb{R}^{d}} e^{-\frac{y \cdot \bar{\sigma}^{-2} y}{2}} f(y) d y,
$$

with the nondegenerate covariance matrix $\bar{\sigma}^{2}$ given in (19).

REMARKS. Theorem 1 readily extends to all finite dimensional marginals of the diffusively scaled process $t \mapsto T^{-1 / 2} X(T t)$, as $T \rightarrow \infty$. In order to spare notation and space we do not make explicit this straightforward extension. The tightness [in $D\left(\mathbb{R}_{+} \rightarrow \mathbb{R}^{d}\right)$ ] of the sequence of diffusively scaled processes $t \mapsto$ $X(T t) / \sqrt{T}$, as $T \rightarrow \infty$, requires some extra arguments, subject of future work.

The concept of harmonic coordinates originates in the seminal papers [16, 24, 25]. This approach was further developed in [17] where the problem of random walks in doubly stochastic random environment was exposed. As pointed out in later works (see, e.g., [5, 26] or [15]), beside the highly innovative ideas in [17] some key arguments are not fully complete there.

The particular case when the jump probabilities admit a bounded cyclic representation is covered by the result of [9]. This condition implies, however, the restriction to $h \in \mathcal{L}^{\infty}$.

By restricting to $p_{k}(\omega)=p_{-k}\left(\tau_{k} \omega\right)$ (i.e., $\left.p_{k}=s_{k}, v_{k} \equiv 0\right)$ [see (5)], the case of random walks among bounded and elliptic random conductances is covered. This is Theorem 1 in [26]. However, since the ellipticity condition (8) is essential in our current setup, Theorem 2.1 of [26] and the main results of [1, 4, 6] are not covered as special cases.

Relaxing the ellipticity condition (8) within this context remains open. This might be possibly resolved by combining ideas and techniques from $[1,6]$ with those in this paper.

For a more exhaustive list of comments on the historical context of the problem and results of this paper, see also [27].

Before turning to the proofs we summarize what is truly new-compared with earlier works on quenched CLT for RWRE_-in the details that follow.

The proof of Proposition 1 relies on an extension of Nash's celebrated moment bound (cf. [23]), to nonreversible, divergence-free drift (i.e., incompressible flow) context, with unbounded $\left(\mathcal{L}^{2+\varepsilon}\right)$ stream tensor. To our knowledge, this is the first such kind of extension of Nash's arguments. 
In the proof of Proposition 2, the construction of the harmonic coordinates is done by functional analytic tools, relying on the the method of relaxed sector condition (cf. [14, 19]), which differs essentially from the methods employed in the cited earlier works.

Some variant of Proposition 4 is a key argument in all proofs of quenched CLT; see, for example, [1, 4, 6, 26]. However, in all these works considerably heavier tools are used in the proof than the soft, merely ergodic arguments of Lemma 4 below.

\section{Proofs.}

3.1. Tightness: Proof of Proposition 1. We follow Nash's blueprint; cf. [23]. See also [3] for a streamlined version of the proof and [2, 6] for adaptation of details to lattice walk on $\mathbb{Z}^{d}$ (rather than continuous diffusion on $\mathbb{R}^{d}$ ) setup, in the context of random walks among random conductances. However, new elements are needed due to the nonreversible drift term. These new elements of the proof will be highlighted.

The main ideas of [23] have been employed in the context of random walks among random conductances; cf. [2, 6]. In all cited works, however, the diffusions and random walks considered have been reversible with respect to uniform measure on $\mathbb{R}^{d}$, respectively, $\mathbb{Z}^{d}$. That is, the diffusion generators in [23] and [3] are purely elliptic in divergence form, the random walks in [2] and [6] are defined by conductances of unoriented edges. It has been well known that the diagonal heat kernel upper bound, (23) below, follows from Nash's inequality not only in the reversible but also in the doubly stochastic/divergence-free cases. The novelty in Proposition 1 and its forthcoming proof is the extension of the entropy and entropyproduction bounds of [23] to the nonreversible case, with doubly stochastic jump rates (or, sourceless, divergence-free, incompressible drift field). The main point is, that in this case and under the integrability condition (12) we are able to control the terms coming from the skew-self-adjoint parts, too, by the entropy production. This is by no means straightforward. Without assuming at least the $H_{-1}$ condition (9), this moment bound is simply not valid, even in the annealed setup. The stronger integrability condition imposed on the stream tensor may well be a technical nuisance only.

All constants in the forthcoming estimates will depend on $d, s_{*}, s^{*}, \varepsilon$ and $h^{*}$ only. See (8) and (13) for their definition. We will adopt the following notational convention: those constants where their being positive (but possibly small) is important will be denoted by lower case symbols $c_{j}$, those ones where their being finite (but possibly large) is the point that will be denoted by upper case symbols $C_{j}$. We will be explicit about which constants depend on which of the parameters listed above. 
We are in the quenched setup. However, in order to lighten notation dependence on $\omega \in \Omega$ will not be shown explicitly within this proof. Denote

$$
\begin{aligned}
q(t, x)=q(t, x, \omega) & :=\mathbf{P}_{\omega}(X(t)=x), \\
M(t)=M(t, \omega) & :=\mathbf{E}_{\omega}(|X(t)|)=\sum_{x \in \mathbb{Z}^{d}}|x| q(t, x), \\
H(t)=H(t, \omega) & :=-\sum_{x \in \mathbb{Z}^{d}} \log q(t, x) q(t, x) .
\end{aligned}
$$

The ingredients of the proof of Proposition 1 are collected in Lemmas 1, 2 and 3 below. For the proofs of Lemmas 1 and 2 we refer to earlier works, [2, 3, 6, 23]. We present full proof of Lemma 3 which contains the new elements.

LEMMA 1. There exists a constant $c_{1}=c_{1}(d) \in(0, \infty)$ such that for any $t>0$ it holds that if $M(t)>1$ then

$$
M(t) \geq c_{1} e^{\frac{H(t)}{d}}
$$

The bound (21) is a direct consequence of the entropy inequality and it is actually valid for any probability distribution $q(x)$ on $\mathbb{Z}^{d}$. See $[3,23]$ for a proof for absolutely continuous probability measures on $\mathbb{R}^{d}$ and $[2,6]$ for its adaptation to probability measures on $\mathbb{Z}^{d}$. We do not reproduce these details here. It is interesting to note that in [23] Nash attributes this particular argument to Carleson.

LEMMA 2. There exists a constant $C_{2}=C_{2}\left(d, s_{*}\right) \in(0, \infty)$ such that

$$
\frac{H(t)}{d} \geq \frac{1}{2} \log t-C_{2} \text {. }
$$

From Nash's inequality it follows, that there exists a constant $C=C\left(d, s_{*}\right)$ such that for all $t \geq 0$ and $x \in \mathbb{Z}^{d}$,

$$
q(t, x) \leq C t^{-d / 2} \text {. }
$$

See Proposition 3 in [19] for an alternative derivation using "evolving sets" method of [22]. We omit the details. The bound (22) follows directly from (23) and the definition of the entropy $H(t)$.

Defining now

$$
G(t):=\frac{H(t)}{d}-\frac{1}{2} \log t+C_{2} \geq 0,
$$

the entropy bound (21) reads

$$
t^{-1 / 2} M(t) \geq c_{3} e^{G(t)},
$$

with $c_{3}=c_{3}\left(d, s_{*}\right)=c_{1} e^{-C_{2}} \in(0, \infty)$. 
LEMMA 3. There exists a constant $C_{4}=C_{4}\left(d, s_{*}, s^{*}, h^{*}\right) \in(0, \infty)$ so that for $\pi$-almost all $\omega \in \Omega$ there exists $t^{*}(\omega)<\infty$ such that for $t>t^{*}(\omega)$,

$$
t^{-1 / 2} M(t) \leq C_{4}\left(G(t)+\varepsilon^{-1}\right)^{\frac{1+\varepsilon}{2+\varepsilon}}
$$

where $\varepsilon>0$ is from (12).

REMARK. Letting $\varepsilon \rightarrow 0, h^{*}=h^{*}(\varepsilon)$ decreases to $\sum_{k l \in \mathcal{E}}\left\|h_{k, l}\right\|_{2}<\infty$. Therefore, $C_{4}$ also decreases to a finite positive limit. Nonetheless, the right-hand side of (25) blows up due to the $\varepsilon^{-1}$ term. This is the reason of imposing (12), with $\varepsilon>0$.

ProOf of Lemma 3. Within this proof we will use the notation

$$
s_{k}(x):=s_{k}\left(\tau_{x} \omega\right), \quad v_{k}(x):=v_{k}\left(\tau_{x} \omega\right), \quad h_{k, l}(x):=h_{k, l}\left(\tau_{x} \omega\right) .
$$

We will use repeatedly Kolmogorov's forward equation for the distribution $q_{t}$ :

$$
\begin{aligned}
\dot{q}(t, x)= & \sum_{x \in \mathbb{Z}^{d}, k \in \mathcal{E}} s_{k}(x)(q(t, x+k)-q(t, x)) \\
& -\sum_{x \in \mathbb{Z}^{d}, k \in \mathcal{E}} v_{k}(x) q(t, x+k) .
\end{aligned}
$$

In the last term, the divergence-freeness (6) of $v$ is used.

First, we provide a lower bound on $\dot{H}(t)$ :

$$
\begin{aligned}
\dot{H}(t)= & \frac{1}{2} \sum_{x \in \mathbb{Z}^{d}, k \in \mathcal{E}} s_{k}(x)(q(t, x+k)-q(t, x))(\log q(t, x+k)-\log q(t, x)) \\
& -\frac{1}{2} \sum_{x \in \mathbb{Z}^{d}, k \in \mathcal{E}} v_{k}(x)(q(t, x+k)+q(t, x))(\log q(t, x+k)-\log q(t, x)) \\
& +\sum_{x \in \mathbb{Z}^{d}, k \in \mathcal{E}} v_{k}(x)(q(t, x+k)-q(t, x)) \\
(27)= & \frac{1}{2} \sum_{x \in \mathbb{Z}^{d}, k \in \mathcal{E}} s_{k}(x)(q(t, x+k)-q(t, x)) \int_{q(t, x)}^{q(t, x+k)} \frac{1}{u} d u \\
& -\frac{1}{2} \sum_{x \in \mathbb{Z}^{d}, k \in \mathcal{E}} v_{k}(x) \int_{q(t, x)}^{q(t, x+k)} \frac{q(x)+q t(x+k)-2 u}{u} d u \\
\geq & s_{*} \sum_{x \in \mathbb{Z}^{d}, k \in \mathcal{E}} \int_{\min \{q(t, x), q(t, x+k)\}}^{\max \{q(t, x), q(t, x+k)\}} \frac{u-\min \{q(t, x), q(t, x+k)\}}{u} d u \\
\geq & c_{5} \sum_{x \in \mathbb{Z}^{d}, k \in \mathcal{E}}\left|\frac{q(t, x+k)-q(t, x)}{q(t, x+k)+q(t, x)}\right|^{2} q(t, x) .
\end{aligned}
$$


The first step follows from from (26) by explicit computations, using the symmetries (5) of $s$ and $v$, and also the divergence-freeness of $v,(6)$. Note that due to this latter the third sum on the right-hand side vanishes. We included it as a dummy. The second step is just transcription of differences to appropriate integrals. In the third step, we have used $s_{k} \geq \max \left\{s_{*},\left|v_{k}\right|\right\}$. Finally, in the last step we have used the bound

$$
b:=\inf _{1<\beta<\infty} \frac{\beta+1}{(\beta-1)^{2}} \int_{1}^{\beta} \frac{u-1}{u} d u=0.8956 \cdots>0,
$$

and got $c_{5}=c_{5}\left(s_{*}\right)=b s_{*}$. Note, that the lower bound on entropy production in terms of Fisher-entropy, (27), looks formally the same as in the reversible case. However, deriving it, one has to control the skew-symmetric part by the symmetric part of the entropy production. This can be done due to incompressibility (or sourcelessness, or divergence-freeness) of the flow $v$.

Next we compute $\dot{M}(t)$ :

$$
\begin{aligned}
\dot{M}(t)= & \frac{1}{2} \sum_{x \in \mathbb{Z}^{d}, k \in \mathcal{E}} s_{k}(x)(|x|-|x+k|)(q(t, x+k)-q(t, x)) \\
& -\frac{1}{2} \sum_{x \in \mathbb{Z}^{d}, k \in \mathcal{E}} v_{k}(x)(|x|+|x+k|)(q(t, x+k)-q(t, x)) \\
= & \frac{1}{2} \sum_{x \in \mathbb{Z}^{d}, k \in \mathcal{E}} s_{k}(x)(|x|-|x+k|)(q(t, x+k)-q(t, x)) \\
& -\frac{1}{2} \sum_{x \in \mathbb{Z}^{d}, k, l \in \mathcal{E}} h_{k, l}(x)(|x+k|-|x+l|)(q(t, x+k+l)-q(t, x)) .
\end{aligned}
$$

The first step follows from (26) by explicit computation, using the symmetries (5) of $s$ and $v$. The second step follows from (11) and the symmetries (10). Hence,

$$
|\dot{M}(t)| \leq C_{6} \sum_{x \in \mathbb{Z}^{d}, k \in \mathcal{E}}\left(s^{*}+\sum_{l \in \mathcal{E}}\left|h_{k, l}(x)\right|\right)\left|\frac{q(t, x+k)-q(t, x)}{q(t, x+k)+q(t, x)}\right| q(t, x),
$$

with $C_{6}=C_{6}(d)$.

Integrating over $t$ and applying Hölder's inequality we obtain

$$
\begin{aligned}
|M(t)| \leq & C_{6} t^{\frac{1}{2+\varepsilon}}\left(\frac{1}{t} \int_{0}^{t} \sum_{x \in \mathbb{Z}^{d}, k \in \mathcal{E}}\left(s^{*}+\sum_{l \in \mathcal{E}}\left|h_{k, l}(x)\right|\right)^{2+\varepsilon} q(u, x) d u\right)^{\frac{1}{2+\varepsilon}} \\
& \times\left(\int_{0}^{t} \sum_{x \in \mathbb{Z}^{d}, k \in \mathcal{E}}\left|\frac{q(u, x+k)-q(u, x)}{q(u, x+k)+q(u, x)}\right|^{\frac{2+\varepsilon}{1+\varepsilon}} q(u, x) d u\right)^{\frac{1+\varepsilon}{2+\varepsilon}} .
\end{aligned}
$$


Due to the (Hopf-) Chacon-Ornstein ergodic theorem (see $[8,12,13]$ or [20]) and integrability of $\left|h_{k, l}\right|^{2+\varepsilon}$ the middle factor in (28) converges to a finite deterministic value, for $\pi$-almost all $\omega \in \Omega$, as $t \rightarrow \infty$. Indeed,

$$
\begin{aligned}
\frac{1}{t} \int_{0}^{t} & \sum_{x \in \mathbb{Z}^{d}, k \in \mathcal{E}}\left(s^{*}+\sum_{l \in \mathcal{E}}\left|h_{k, l}(x)\right|\right)^{2+\varepsilon} q(u, x) d u \\
= & \frac{1}{t} \int_{0}^{t} \sum_{k \in \mathcal{E}} \mathbf{E}_{\omega}\left(\left(s^{*}+\sum_{l \in \mathcal{E}}\left|h_{k, l}(\eta(u))\right|\right)^{2+\varepsilon}\right) d u,
\end{aligned}
$$

where $t \mapsto \eta(t)$ is the Markov process of the environment seen by the random walker, defined in (3), which is stationary and ergodic on $(\Omega, \pi)$. This is the typical context for the (Hopf-) Chacon-Ornstein theorem. The right-hand side of (29) $\pi$ almost-surely converges to

$$
C_{7}^{2+\varepsilon}:=\sum_{k \in \mathcal{E}} \int_{\Omega}\left(s^{*}+\sum_{l \in \mathcal{E}}\left|h_{k, l}(\omega)\right|\right)^{2+\varepsilon} d \pi(\omega)<\infty .
$$

Obviously, $C_{7}=C_{7}\left(d, s^{*}, h^{*}\right)$. Note that this is the only argument where the stronger integrability condition (12) is used.

On the other hand, due to (27) and a Hölder bound, the last factor in (28) is dominated by the entropy production. Altogether we obtain that for $\pi$-almost all $\omega \in \Omega$, there exists $t^{*}(\omega)<\infty$, such that for all $t>t^{*}(\omega)$,

$$
|M(t)| \leq C_{8} t^{\frac{1}{2+\varepsilon}}\left(\int_{0}^{t} \dot{H}(u)^{\frac{2+\varepsilon}{2+2 \varepsilon}} d u\right)^{\frac{1+\varepsilon}{2+\varepsilon}}
$$

where $C_{8}=C_{8}\left(d, s_{*}, s^{*}, h^{*}\right):=2 C_{6} C_{7} c_{5}^{-1 / 2}$.

The rest is straight sailing. Following $[3,23]$ (see also $[2,6]$ ), with due modifications,

$$
\begin{aligned}
\int_{0}^{t} \dot{H} & (u)^{\frac{2+\varepsilon}{2+2 \varepsilon}} d u \\
& =\int_{0}^{t}\left(\dot{G}(u)+\frac{1}{2 u}\right)^{\frac{2+\varepsilon}{2+2 \varepsilon}} d u \\
& =\int_{0}^{t}(2 u)^{-\frac{2+\varepsilon}{2+2 \varepsilon}}(1+2 u \dot{G}(u))^{\frac{2+\varepsilon}{2+2 \varepsilon}} d u \\
& \leq \int_{0}^{t}\left((2 u)^{-\frac{2+\varepsilon}{2+2 \varepsilon}}+\frac{2+\varepsilon}{2+2 \varepsilon}(2 u)^{\frac{\varepsilon}{2+2 \varepsilon}} \dot{G}(u)\right) d u \\
& =\frac{1+\varepsilon}{\varepsilon}(2 t)^{\frac{\varepsilon}{2+2 \varepsilon}}+\frac{2+\varepsilon}{2+2 \varepsilon}(2 t)^{\frac{\varepsilon}{2+2 \varepsilon}} G(t)-\frac{\varepsilon}{2+2 \varepsilon} \int_{0}^{t}(2 u)^{-\frac{2+\varepsilon}{2+2 \varepsilon}} G(u) d s \\
& \leq 3 t^{\frac{\varepsilon}{2+2 \varepsilon}}\left(\varepsilon^{-1}+G(t)\right)
\end{aligned}
$$

Inserting this into (30) we obtain (25) of Lemma 3, with $C_{4}=3 C_{8}$. 
To conclude the proof of Proposition 1 note that (24) and (25) jointly imply that there exists a constant $C_{9}=C_{9}\left(\varepsilon, d, s_{*}, s^{*}, h^{*}\right)$ so that for $\pi$-almost all $\omega \in \Omega$, there exists a $t^{*}(\omega)$ so that for $t>t^{*}(\omega), G(t) \leq C_{9}$. Hence follows (14), via (25).

3.2. Some operators over $\mathcal{H}$ and $\mathcal{G}$. First, we recall from [19] some bounded operators acting on the Hilbert spaces $\mathcal{H}$ and $\mathcal{G}$.

Let $\left(\Omega, \mathcal{F}, \pi, \tau_{z}: z \in \mathbb{Z}^{d}\right)$ be a probability space with an is ergodic $\mathbb{Z}^{d}$-action. The gradient, Laplacian and Riesz operators are all directly expressed with the help of the shift operators $U_{k} f(\omega):=f\left(\tau_{k} \omega\right)$, as follows:

$\nabla_{k}, \Delta, \Gamma_{k}: \mathcal{H} \rightarrow \mathcal{H}:$

$$
\nabla_{k}:=U_{k}-I, \quad \Delta:=2 \sum_{k \in \mathcal{E}} \nabla_{k}, \quad \Gamma_{k}:=|\Delta|^{-1 / 2} \nabla_{k} .
$$

$\nabla, \Gamma: \mathcal{H} \rightarrow \mathcal{G}:$

$$
(\nabla f)_{k}:=\nabla_{k} f, \quad(\Gamma f)_{k}:=\Gamma_{k} f
$$

$\nabla^{*}, \Gamma^{*}: \mathcal{G} \rightarrow \mathcal{H}:$

$$
\nabla^{*} g:=\sum_{k \in \mathcal{E}} \nabla_{-k} g_{k}, \quad \Gamma^{*} g:=\sum_{k \in \mathcal{E}} \Gamma_{-k} g_{k} .
$$

The following identities hold:

$$
\nabla^{*} \nabla=-\Delta, \quad \Gamma^{*} \Gamma=I_{\mathcal{H}}, \quad \Gamma \Gamma^{*}=I_{\mathcal{G}} .
$$

The first two follow directly from the definitions and straightforward computations. The proof of the third one relies on $\operatorname{Ker}\left(\nabla^{*}\right)=\operatorname{Ker}\left(\Gamma^{*}\right)=\left\{0_{\mathcal{G}}\right\}$. This follows from ergodicity, $\operatorname{Ker}(\Delta)=\left\{0_{\mathcal{H}}\right\}$ and its proof is left as an exercise. The last two identities in (32) mean that $\Gamma: \mathcal{H} \rightarrow \mathcal{G}$ is an isometric isomorphism. This fact will have importance below.

We will also use the multiplication operators $M_{k}, N_{k}: \mathcal{L}^{2}(\Omega, \pi) \rightarrow \mathcal{L}^{2}(\Omega, \pi)$, $k \in \mathcal{E}$ [see (KT38), (KT39)]:

$$
N_{k} f(\omega):=\left(s_{k}(\omega)-s_{*}\right) f(\omega), \quad M_{k} f(\omega):=v_{k}(\omega) f(\omega),
$$

and recall the commutation relations (KT40):

$$
\begin{aligned}
-\sum_{k \in \mathcal{E}} N_{k} \nabla_{k} & =-\sum_{k \in \mathcal{E}} \nabla_{-k} N_{k}=\frac{1}{2} \sum_{k \in \mathcal{E}} \nabla_{-k} N_{k} \nabla_{k}=: T=T^{*} \geq 0, \\
\sum_{k \in \mathcal{E}} M_{k} \nabla_{k} & =-\sum_{k \in \mathcal{E}} \nabla_{-k} M_{k}=: A=-A^{*},
\end{aligned}
$$

which follow directly from (5) and (6).

Strictly speaking, the multiplication operators $M_{k}$ and $N_{k}$ do not preserve the subspace $\mathcal{H} \subset \mathcal{L}^{2}(\Omega, \pi)$ of zero mean elements. However, they only appear in the combinations $\sum_{k \in \mathcal{E}} N_{k} \nabla_{k}$, respectively, $\sum_{k \in \mathcal{E}} M_{k} \nabla_{k}$, which due to the commutation relations (34) do preserve $\mathcal{H}$. 
Also recall the decomposition of the infinitesimal generator $L$ of the environment process $t \mapsto \eta(t)$ into self-adjoint and skew-self-adjoint parts [cf. (KT41)(KT43)]:

$$
L=\frac{1}{2} \Delta-T+A=-S+A .
$$

Note that the (absolute value) of the Laplacian minorizes and majorizes the selfadjoint part of the infinitesimal generator:

$$
s_{*}|\Delta| \leq 2 S \leq s^{*}|\Delta| \text {. }
$$

The inequalities are meant in operator sense. The ellipticity condition (8) is used in the lower bound, and bounded jump rates in the upper bound.

3.3. Harmonic coordinates: Proof of Proposition 2. Since $\Gamma: \mathcal{H} \rightarrow \mathcal{G}$ is an isometric isomorphism [see (32)], we can assume that

$$
\theta=\Gamma \chi,
$$

with some $\chi \in \mathcal{H}$, and write the equation (15) for $\chi \in \mathcal{H}$ as follows:

$$
\left(|\Delta|^{1 / 2}+\sum_{k \in \mathcal{E}} N_{k} \Gamma_{k}+\sum_{k \in \mathcal{E}} M_{k} \Gamma_{k}\right) \chi=\phi .
$$

This is the equation to be solved for $\chi \in \mathcal{H}$.

In order to present the argument in its most transparent form, let us first make the simplifying assumption that the symmetric part $s_{k}$ of the jump rates $p_{k}$ [see (KT5)] are actually constant, $s_{k}(\omega) \equiv 1 \pi$-a.s.:

$$
p_{k}(\omega)=1+v_{k}(\omega) \text {. }
$$

This is the case treated in an early version of [19], available at arxiv [18]. Its advantage is that the relevant ideas appear in their most transparent form, without the formal (but unessential) complications caused by the nonconstant symmetric parts. In this case we have [see (33)]

$$
N_{k}=0 \quad \text { for all } k \in \mathcal{E} \text {. }
$$

Thus (36) reduces to

$$
\left(|\Delta|^{1 / 2}+\sum_{k \in \mathcal{E}} M_{k} \Gamma_{k}\right) \chi=\phi .
$$

Since it is assumed that $\phi \in \mathcal{H}_{-1}$ we can multiply equation (38) from the left by $|\Delta|^{-1 / 2}$ to get

$$
\left(I+|\Delta|^{-1 / 2} \sum_{k \in \mathcal{E}} M_{k} \Gamma_{k}\right) \chi=|\Delta|^{-1 / 2} \phi .
$$


On the left-hand side of this equation we have exactly the densely defined and closed unbounded operator

$$
-B^{*}:=|\Delta|^{-1 / 2} \sum_{k \in \mathcal{E}} M_{k} \Gamma_{k}
$$

[see (KT58)] which in Proposition 2 of [18] is proved to be skew-self-adjoint (not merely the adjoint of a skew-symmetric one). Recall that this is the key technical point in the proof of the main result in [18]. Thus, the spectrum of the operator $B=-B^{*}$ is on the imaginary axis and, therefore, on the left- hand side of (39) $I-B^{*}=I+B$ is invertible, the unique solution of (38) being

$$
\chi=(I+B)^{-1}\left(|\Delta|^{-1 / 2} \phi\right) \text {. }
$$

Finally,

$$
\theta_{k}=\Gamma_{k}(I+B)^{-1}\left(|\Delta|^{-1 / 2} \phi\right), \quad k \in \mathcal{E} .
$$

These are bona fide elements of $\mathcal{H}$ since

$$
|\Delta|^{-1 / 2} \phi \in \mathcal{H}, \quad\left\|(I+B)^{-1}\right\| \leq 1, \quad\left\|\Gamma_{k}\right\| \leq 1 .
$$

Now we go to the general case, without assuming (37). It is proved in Theorem RSC2 of [19] that due to (35) the operator $|\Delta|^{1 / 2} S^{-1 / 2}$ is bounded and has a bounded inverse and the a priori densely defined operator $C:=S^{-1 / 2} A S^{-1 / 2}$ is essentially skew-self-adjoint. (See the proof of Theorem RSC2 in the Appendix of [19].) Recall that this is the key technical point in the proof of the main result of [19]. Hence it follows that

$$
\chi:=\left(|\Delta|^{1 / 2} S^{-1 / 2}\right)(I+C)^{-1}\left(S^{-1 / 2}|\Delta|^{1 / 2}\right)|\Delta|^{-1 / 2} \phi
$$

is a bona fide element of $\mathcal{H}$. Indeed, due to (9) $|\Delta|^{-1 / 2} \phi \in \mathcal{H}$ and

$$
\left\|S^{-1 / 2}|\Delta|^{1 / 2}\right\|=\left\||\Delta|^{1 / 2} S^{-1 / 2}\right\|<\infty, \quad\left\|(I+C)^{-1}\right\| \leq 1 .
$$

It is an easy formal computation to check that $\chi$ in (40) provides the solution to the equation (36) in the general case, and hence

$$
\theta_{k}=\Gamma_{k}\left(|\Delta|^{1 / 2} S^{-1 / 2}\right)(I+C)^{-1}\left(S^{-1 / 2}|\Delta|^{1 / 2}\right)|\Delta|^{-1 / 2} \phi .
$$

3.4. Martingale CLT: Proof of Proposition 3 and Corollary 1. This follows from the most conventional application of the martingale central limit theorem; see, for example, $[10,11]$. Due to the choice of $\theta$, for $\pi$-a.a. $\omega \in \Omega$ the quenched process $t \mapsto Y(t)$ defined in (17) is a martingale. Its infinitesimal conditional variance process is

$$
\lim _{h \rightarrow 0} h^{-1} \mathbf{E}_{\omega}\left((Y(t+h)-Y(t))^{2} \mid \mathcal{F}_{t}\right)=\sigma^{2}(\eta(t)),
$$


where $t \mapsto \eta(t):=\tau_{X(t)} \omega$ is the environment process as seen by the random walk, defined in (3), and $\sigma^{2}: \Omega \rightarrow \mathbb{R}_{+}$is

$$
\sigma^{2}(\omega)=\sum_{k \in \mathcal{E}} p_{k}(\omega)\left|\theta_{k}(\omega)\right|^{2}
$$

The key observation is that since the Markov process $t \mapsto \eta(t)$ is stationary and ergodic (see Section 1.2 of [19]) the following strong law of large numbers holds:

$$
\lim _{t \rightarrow \infty} \frac{1}{t} \int_{0}^{t} \sigma^{2}(\eta(s)) d s=\int_{\Omega} \sigma^{2}(\omega) d \pi(\omega)=: \bar{\sigma}^{2}, \quad \pi \text {-a.s. }
$$

Positivity of the variance $\bar{\sigma}^{2}$ follows from the the (skew)symmetry of $v$ in the second line of (5) and the ellipticity condition (8). Indeed, from these relations it follows that

$$
\begin{aligned}
\bar{\sigma}^{2} & =\sum_{k \in \mathcal{E}} \int_{\Omega} p_{k}(\omega)\left|\theta_{k}(\omega)\right|^{2} d \pi(\omega)=\sum_{k \in \mathcal{E}} \int_{\Omega} s_{k}(\omega)\left|\theta_{k}(\omega)\right|^{2} d \pi(\omega) \\
& \geq s_{*} \sum_{k \in \mathcal{E}} \int_{\Omega}\left|\theta_{k}(\omega)\right|^{2} d \pi(\omega)>0 .
\end{aligned}
$$

In the middle equality, the symmetries (5) are used. This concludes the proof of Proposition 3.

Corollary 1 follows directly. We apply the standard martingale decomposition [see (KT25)] and Proposition 3:

$$
Y^{*}(t)=\left(X(t)-\int_{0}^{t} \phi^{*}\left(\tau_{X(s)} \omega\right) d s\right)+\left(\int_{0}^{t} \phi^{*}\left(\tau_{X(s)} \omega\right) d s-\Theta^{*}(\omega, X(t))\right),
$$

and note that the martingale CLT applies. The expression (19) of the asymptotic covariance matrix follows as above.

3.5. Asymptotically vanishing corrector: Proof of Proposition 4. We write [like in (KT74)]

$$
\begin{aligned}
\mathbf{P}_{\omega}(\mid & \Psi(X(t)) \mid>\delta \sqrt{t}) \\
& \leq \mathbf{P}_{\omega}(\{|\Psi(X(t))|>\delta \sqrt{t}\} \cap\{|X(t)| \leq K \sqrt{t}\})+\mathbf{P}_{\omega}(|X(t)|>K \sqrt{t}) \\
& \leq \delta^{-1} t^{-1 / 2} \mathbf{E}_{\omega}\left(|\Psi(X(t))| \mathbb{1}_{\{|X(t)| \leq K \sqrt{t}\}}\right)+K^{-1} t^{-1 / 2} \mathbf{E}_{\omega}(|X(t)|) .
\end{aligned}
$$

Using the diagonal heat kernel upper bound (23) in the first term and the moment bound (14) in the second term from here we readily obtain

$$
\begin{aligned}
& \varlimsup_{t \rightarrow \infty} \mathbf{P}_{\omega}(|\Psi(X(t))|>\delta \sqrt{t}) \\
& \leq C \delta^{-1} \varlimsup_{t \rightarrow \infty} t^{-(1+d) / 2} \sum_{|x| \leq K \sqrt{t}}|\Psi(x)|+M^{*} K^{-1} .
\end{aligned}
$$

The statement of Proposition 4, (20), will follow from the following strong law of large numbers. 
LEMMA 4. Let $\left(\Omega, \mathcal{F}, \pi, \tau_{z}: z \in \mathbb{Z}^{d}\right)$ be a probability space with an ergodic $\mathbb{Z}^{d}$-action and $\Omega \times \mathbb{Z}^{d} \ni x \mapsto \Psi(\omega, x) \in \mathbb{R}$ be a zero-mean $\mathcal{L}^{2}$-cocycle. Then

$$
\lim _{N \rightarrow \infty} N^{-(d+1)} \sum_{|x| \leq N}|\Psi(x)|=0, \quad \pi \text {-a.s. }
$$

Remarks on Lemma 4. The statement Lemma 4 holds true for zero-mean $\mathcal{L}^{p}$ cocycles, with $p>1$. However, here we only need the $\mathcal{L}^{2}$ version.

The weaker statement

$$
\lim _{N \rightarrow \infty} N^{-d} \sum_{|x| \leq N} \mathbb{1}_{\{|\Psi(x)|>\varepsilon N\}}=0, \quad \pi \text {-a.s., } \forall \varepsilon>0,
$$

readily follows from (42) by Markov's inequality.

Various versions of (42) or (43) appear as key ingredient in all proofs of quenched CLT for random walks among random conductances. As examples (in chronological order), see (0.13) (1.23) in [26]; (5.15) in [4]; (2.15) and (5.25) in [6]; (7.17) in [21]; (12) in [1]; (4.1) in [7]. (The list is certainly not exhaustive.) However, it seems to be the case that in all these works heavier tools had been used than the merely ergodic arguments employed in the proof below. This is our reason to include it here.

ProOF OF LEMma 4. We will prove the lemma by induction on the dimension $d$ and for the sequence of cubic boxes $[0, N-1]^{d}$ rather than $[-N, N]^{d}$. For $d=1$, the statement of the lemma is a direct consequence of Birkhoff's ergodic theorem. We will use the notation $(\underline{n}, m) \in[0, N-1]^{d} \times[0, N-1]$. Fix $L<\infty$ and write

$$
\begin{aligned}
& \sum_{\underline{n} \in[0, N-1]^{d}} \sum_{m \in[0, N-1]}|\Psi(\underline{n}, m)| \\
& \leq \sum_{\underline{n} \in[0, N-1]^{d}} \sum_{l=0}^{L-1} \sum_{j=0}^{L(N-1) / L\rfloor}|\Psi(\underline{n}, l+j L)| \\
& \leq N \sum_{\underline{n} \in[0, N-1]^{d}}|\Psi(\underline{n}, 0)|+\frac{N}{L} \sum_{\underline{n} \in[0, N-1]^{d}} \sum_{l=0}^{L-1}|\Psi(\underline{n}, l)-\Psi(\underline{n}, 0)| \\
& +\sum_{\underline{n} \in[0, N-1]^{d}} \sum_{l=0}^{L-1} \sum_{j=1}^{L(N-1) / L\rfloor} \sum_{i=0}^{j-1}|\Psi(\underline{n}, l+(i+1) L)-\Psi(\underline{n}, l+i L)| .
\end{aligned}
$$

By the induction hypothesis, for the first term we get

$$
\lim _{N \rightarrow \infty} N^{-(d+2)} N \sum_{\underline{n} \in[0, N-1]^{d}}|\Psi(\underline{n}, 0)|=\lim _{N \rightarrow \infty} N^{-(d+1)} \sum_{\underline{n} \in[0, N-1]^{d}}|\Psi(\underline{n}, 0)|=0 .
$$


For the second term we apply directly the multidimensional version of the almost sure ergodic theorem:

$$
\begin{aligned}
\lim _{N \rightarrow \infty} & N^{-(d+2)} \frac{N}{L} \sum_{\underline{n} \in[0, N-1]^{d}} \sum_{l=0}^{L-1}|\Psi(\underline{n}, l)-\Psi(\underline{n}, 0)| \\
& =L^{-1} \sum_{l=0}^{L-1} \lim _{N \rightarrow \infty} N^{-d-1} \sum_{\underline{n} \in[0, N-1]^{d}}|\Psi(\underline{n}, l)-\Psi(\underline{n}, 0)|=0 .
\end{aligned}
$$

Finally, we turn to the third term on the right-hand side of (44):

$$
\begin{aligned}
\varlimsup_{N \rightarrow \infty} & N^{-(d+2)} \sum_{\underline{n} \in[0, N-1]^{d}} \sum_{l=0}^{L-1} \sum_{j=1}^{\lfloor(N-1) / L\rfloor} \sum_{i=0}^{j-1}|\Psi(\underline{n}, l+(i+1) L)-\Psi(\underline{n}, l+i L)| \\
\leq & \frac{1}{L} \sum_{l=0}^{L-1} \lim _{N \rightarrow \infty} \frac{L^{2}}{N^{2}} \sum_{j=1}^{\lfloor(N-1) / L\rfloor} j \frac{1}{N^{d} j} \\
& \times \sum_{\underline{n} \in[0, N-1]^{d}} \sum_{i=0}^{j-1} \frac{|\Psi(\underline{n}, l+(i+1) L)-\Psi(\underline{n}, l+i L)|}{L} \\
= & L^{-1} \mathbf{E}(|\Psi(\underline{0}, L)-\Psi(\underline{0}, 0)|) .
\end{aligned}
$$

In the second step we have applied the multidimensional unrestricted almost sure ergodic theorem; see Theorem 6.1 .2 of [20].

Finally, letting $L \rightarrow \infty$, by the multidimensional version of the mean ergodic theorem we obtain (42) in dimension $d+1$.

Going now back to (41), first applying (42) and then letting $K \rightarrow \infty$ we obtain (20).

Acknowledgements. Thanks are due to Marek Biskup and Takashi Kumagai for insisting on the question of extending the result of [19] to quenched setup and for their helpful remarks on the context of Lemma 1. I also thank Gady Kozma's illuminating comments.

\section{REFERENCES}

[1] Anders, S., Deuschel, J.-D. and SlowiK, M. (2015). Invariance principle for the random conductance model in a degenerate ergodic environment. Ann. Probab. 43 1866-1891. MR3353817

[2] BARlow, M. (2004). Random walks on supercritical percolation clusters. Ann. Probab. 32 3024-3084. MR2094438

[3] BASS, R. F. (2002). On Aronson's upper bounds for heat kernels. Bull. Lond. Math. Soc. 34 415-419. 
[4] BERgER, N. and BisKUP, M. (2007). Quenched invariance principle for simple random walk on percolation clusters. Probab. Theory Related Fields 137 83-120. MR2278453

[5] Biskup, M. (2011). Recent progress on the random conductance model. Probab. Surv. 8294 373.

[6] Biskup, M. and PREscott, T. M. (2007). Functional CLT for random walk among bounded random conductances. Electron. J. Probab. 12 1323-1348.

[7] BISKUP, M. and RodRIGUEZ, P.-F. (2018). Limit theory for random walks in degenerate timedependent random environment. J. Funct. Anal. 274 985-1046.

[8] Chacon, R. V. and Ornstein, D. S. (1960). A general ergodic theorem. Illinois J. Math. 4 $153-160$.

[9] Deuschel, J.-D. and Kösters, H. (2008). The quenched invariance principle for random walks in random environments admitting a bounded cycle representation. Ann. Inst. Henri Poincaré B, Calc. Probab. Stat. 44 574-591.

[10] Hall, P. and Heyde, C. C. (1980). Martingale Limit Theory and Its Application. Academic Press, New York.

[11] Helland, I. S. (1982). Central limit theorems for martingales with discrete or continuous time. Scand. J. Stat. 9 79-94.

[12] Hopf, E. (1954). The general temporally discrete Markoff process. J. Rational Mech. Anal. 3 $13-45$.

[13] Hopf, E. (1960). On the ergodic theorem for positive linear operators. J. Reine Angew. Math. 205 101-106.

[14] Horváth, I., Tóth, B. and Vető, B. (2012). Relaxed sector condition. Bull. Inst. Math. Acad. Sin. (N.S.) 7 463-476.

[15] Komorowski, T., Landim, C. and Olla, S. (2012). Fluctuations in Markov ProcessesTime Symmetry and Martingale Approximation. Grundlehren der Mathematischen Wissenschaften 345. Springer, Berlin.

[16] KozLov, S. M. (1979). The averaging of random operators. Mat. Sb. 109 188-202.

[17] KozLov, S. M. (1985). The method of averaging and walks in inhomogeneous environments. Uspekhi Mat. Nauk 40 61-120.

[18] Kozma, G. and Tóтн, B. (2014). Central limit theorem for random walks in divergence-free random drift field: $H_{-1}$ suffices. Available at http://arxiv.org/abs/1411.4171v1.

[19] KozMA, G. and TóTH, B. (2017). Central limit theorem for random walks in doubly stochastic random environment: $H_{-1}$ suffices. Ann. Probab. 45 4307-4347. MR3737912

[20] Krengel, U. (1985). Ergodic Theorems. De Gruyter Studies in Mathematics 6. de Gruyter, Berlin.

[21] Kumagai, T. (2014). Random Walks on Disordered Media and Their Scaling Limits. In École d'Été de Probabilités de Saint-Flour XL-2010. Lecture Notes in Math. 2101. Springer, New York.

[22] Morris, B. and Peres, Y. (2005). Evolving sets, mixing and heat kernel bounds. Probab. Theory Related Fields 133 245-266.

[23] Nash, J. (1958). Continuity of solutions of parabolic and elliptic equations. Amer. J. Math. 80 931-954.

[24] Osada, H. (1983). Homogenization of diffusion processes with random stationary coefficients. In Lecture Notes in Mathematics 1021 507-517. Springer, Berlin.

[25] Papanicolaou, G. C. and Varadhan, S. R. S. (1981). Boundary value problems with rapidly oscillating random coefficients. In Random Fields, Vol. I, II (Esztergom, 1979). Colloquia Mathematica Societatis János Bolyai 27 835-873. North-Holland, Amsterdam. MR0712714

[26] Sidoravicius, V. and Sznitman, A.-S. (2004). Quenched invariance principles for walks on clusters of percolation or among random conductances. Probab. Theory Related Fields 129 219-244. 
[27] Tо́тн, B. (2018). Diffusive and Super-Diffusive Limits for Random Walks and Diffusions with Long Memory. In Proceedings of the International Congress of Mathematicians-2018. Rio de Janeiro. To appear.

[28] Zeitouni, O. (2004). Random walks in random environment. In Lectures on Probability Theory and Statistics. Lecture Notes in Math. 1837 189-312. Springer, Berlin. MR2071631

SCHOOL OF MATHEMATICS

UNIVERSITY OF BRISTOL

BRISTOL, BS8 1TW

UNITED KINGDOM

AND

RÉNYi INSTITUTE OF MATHEMATICS

HUNGARIAN ACADEMY OF SCIENCES

BUDAPEST, H-1053

HUNGARY

E-MAIL: balint.toth@bristol.ac.uk toth.balint@renyi.mta.hu 